\title{
Avaliação do conhecimento sobre o tratamento fisioterápico da incontinência urinária de esforço entre gestantes e puér- peras atendidas na rede pública de saúde do município de Patrocínio-MG
}

\section{Evaluation of knowledge about physiotherapeutic treatment of stress urinary in- continence in pregnant and postpartum women attended at public health services of Patrocínio-MG}

\author{
Ademar Gonçalves Caixeta Neto ${ }^{1}$, Andréa Caixeta Gonçalves², Lilian Figueiredo Ribas ${ }^{3}$, Juliana \\ Araújo Freitas Silva ${ }^{4}$
}

1- Universidade José do Rosário Vellano, Belo Horizonte, MG, Brasil.

2-Hospital de Clínicas da Universidade Federal de Uberlândia, Uberlândia, MG, Brasil.

3- Hospital Felício Rocho, Belo Horizonte, MG, Brasil.

4- Centro Universitário do Cerrado - Patrocínio, Patrocínio, MG, Brasil.

Objetivo: avaliar o conhecimento a respeito do tratamento fisioterapêutico na incontinência urinária de esforço entre gestantes e puérperas atendidas na rede pública de saúde do município de Patrocínio-MG. Método: foi realizado estudo de delineamento transversal com 80 mulheres ( 40 gestantes e 40 puérperas), com idades entre 16 e 38 anos, selecionadas aleatoriamente, e residentes no município de Patrocínio-MG, após aprovação pelo Comitê de Ética do UNICERP. Os critérios de inclusão adotados foram ter idade mínima de 15 anos e máxima de 40 anos, e estar no último trimestre de gestação ou puerpério imediato. Para verificar o conhecimento sobre a fisioterapia na incontinência urinária foi aplicado um questionário semi-estruturado elaborado pelos pesquisadores. A comparação entre os grupos foi feita por meio do teste do qui-quadrado de Pearson ou exato de Fisher,

Palavras-chave:

Fisioterapia;

Incontinência Urinária de

Esforço;

Gestantes;

Puérperas. adotando-se nível de significância estatística de 95\%. Resultados: entre todas as pacientes $(\mathrm{n}=80)$, $17.5 \%$ das gestantes e $20 \%$ das puérperas sabiam sobre a atuação da fisioterapia no tratamento da incontinência urinária, não havendo diferença significativa entre os grupos $(\mathrm{p}=0,77)$. Nas pacientes com incontinência urinária $(n=27)$, estes números foram de $23.5 \%$ e $20 \%$ para gestantes e puérperas, respectivamente, as quais não diferiram estatisticamente $(\mathrm{p}=0,83)$. Conclusão: foi observado baixo conhecimento sobre a atuação da fisioterapia no tratamento da incontinência urinária de esforço, o qual representa a principal alternativa ao tratamento cirúrgico da mesma.

\section{ABSTRACT}

Objective: the aim of study was to evaluate the knowledge about physiotherapeutic treatment of stress urinary incontinence in pregnant and postpartum women attended at public health services of Patrocínio-MG, Brazil. Method: a cross-sectional study was conducted with 80 women (40 pregnant and 40 postpartum), aged between 16 and 38 years, randomly selected, and residents from Patrocínio-MG, Brazil, after approval by the Ethics Committee of UNICERP. The Inclusion criteria were having minimum age between 15 and 40 years, and be in the last trimester of pregnancy or postpartum period. To assess the knowledge about physiotherapy in urinary incontinence was applied a semi-structured questionnaire developed by the researchers. The comparison between groups was performed using the Pearson chi-square test or Fisher exact test, adopting a significance level of

Keywords:

Physiotherapy;

Urinary Incontinence;

Pregnant Women;

Postpartum Women.
$95 \%$. Results: among all patients $(n=80), 17.5 \%$ of the pregnant and $20 \%$ of the postpartum women knew about the role of physiotherapy in the treatment of urinary incontinence, with no significant difference $(p=0,77)$. In patients with urinary incontinence $(n=27)$, it was equal to $23.5 \%$ and $20 \%$ for pregnant and postpartum women, respectively, without statistically different $(p=0,83)$. Conclusion: it was observed low knowledge about the physiotherapy in the treatment of stress urinary incontinence, which represents the main alternative to the it surgical treatment. 


\section{INTRODUÇÃO}

O termo incontinência de "esforço" foi cunhado inicialmente por Sir Eardley, o qual definiu essa condição como "perda de urina através da uretra intacta, sob certas condições que causam aumento da pressão intra-abdominal". ${ }^{1}$ A Incontinência Urinária de Esforço (IUE) ocorre quando a pressão intra-vesical supera a pressão que o mecanismo de fechamento uretral pode suportar. ${ }^{2}$

É uma situação bastante comum, entre mulheres, particularmente durante e após as gestações, podendo descrever tanto um sintoma quanto um diagnóstico. ${ }^{3}$ Como sintoma, se refere meramente à perda urinária associada a qualquer atividade que provoque o aumento da pressão intra-abdominal, tais como tosse, espirro e/ou realização de outros tipos de esforços. ${ }^{3}$

A prevalência de incontinência urinária na população feminina varia conforme a faixa etária ${ }^{4}$, sendo observadas taxas de aproximadamente 30\% entre adultas 5 e de até $55 \%$ entre idosas. ${ }^{4-7}$ A IUE é a forma mais comum de queixa urinária entre mulheres, seguida pela de urgência ${ }^{8}$, as quais apresentam taxas de $50 \%$ e $20 \%$, respectivamente. ${ }^{4}$

Gestação, paridade, tipo de parto, obesidade, peso do recém-nascido, idade elevada e redução dos níveis de estrógeno pós-menopausa são os principais fatores relacionados à ocorrência de sintomas da IUE. ${ }^{9-13}$

O estudo urodinâmico é o principal método utilizado para o diagnóstico da $\mathrm{IUE}^{14}$, o qual se baseia também na análise da história clínica, realização do exame físico e de testes complementares. ${ }^{15}$

A conduta para o tratamento da IUE, que pode ser cirúrgico ou conservador, baseia-se nos achados clínicos e no grau de desconforto da paciente ${ }^{3}$, visando melhorar a sua qualidade de vida, evitando tanto problemas psicológicos, sociais e higiênicos quanto a ocorrência de infecções do sistema urinário. ${ }^{16,17} \mathrm{O}$ tratamento pode envolver desde o treinamento da bexiga e exercícios para músculos do assoalho pélvico, até a eletroestimulação funcional desta musculatura. ${ }^{18}$

Adespeito doreduzido encaminhamento médico, o tratamento fisioterapêutico para incontinência urinária de esforço vem ganhando cada vez mais espaço e aceitação. ${ }^{18,19}$ Por isso, é de fundamental importância conscientizar as mulheres quanto à necessidade do tratamento, bem como informar sobre seus benefícios, uma vez que o mesmo poderá não apenas contribuir para seu conforto e melhora da sua qualidade de vida, mas também evitar a necessidade de uma eventual cirurgia. ${ }^{19}$

Neste contexto, o objetivo do trabalho foi avaliar o conhecimento a respeito da fisioterapia na IUE entre gestantes e puérperas atendidas na rede pública de saúde do município de Patrocínio-MG.

\section{MÉTODO}

Foi realizado estudo transversal entre os meses de julho e setembro de 2008, no Hospital e Maternidade Santa Casa de Misericórdia e em Unidades Básicas de Saúde (UBS) do município de Patrocínio-MG, com mulheres que estavam no último semestre de gestação ou no puerpério imediato, e idades entre 16 e 38 anos.

O estudo contou inicialmente com 95 mulheres, selecionadas de forma aleatória, sendo a amostra final constituída de 80 mulheres (40 gestantes e 40 puérperas), as quais manifestaram desejo em participar da pesquisa de forma espontânea e assinaram um Termo de Consentimento Livre e Esclarecido (TCLE).

No período descrito anteriormente, foi aplicado um questionário semi-estruturado elaborado pelos pesquisadores para aferir o conhecimento sobre a fisioterapia na IUE entre gestantes e puérperas sintomáticas e assintomáticas.

Os critérios de inclusão adotados foram ter idade mínima de 15 anos e máxima de 40 anos, e estar no $3^{\circ}$ trimestre de gestação ou puerpério imediato.

De acordo com classificação de Moreno $^{20}$ foram consideradas puérperas em pós-parto imediato aquelas mulheres que se encontravam entre o $1^{\circ}$ e o $10^{\circ}$ dia após o parto.

Em relação à análise estatística dos dados foi realizado o teste do qui-quadrado de Pearson ou exato de Fisher para comparação dos grupos, adotando-se nível de significância estatística de 95\%.

O estudo respeitou a resolução $n^{\circ} 466$ do Conselho Nacional de Saúde e foi conduzido após aprovação pelo Comitê de Ética em Pesquisa do Centro Universitário do Cerrado - Patrocínio sob $n^{\circ}$ $12 / 2008$.

\section{RESULTADOS}

Ao analisar o conhecimento acerca da fisioterapia no tratamento da IUE entre todas as participantes do estudo, apenas $17.5 \%$ das gestantes 
e $20 \%$ das puérperas relataram saber sobre o papel do fisioterapeuta na prevenção e reabilitação dos sintomas (tabela 1).

Tabela 1 - Avaliação do conhecimento sobre fisioterapia na IUE entre gestantes e puérperas $(n=80)$.

\begin{tabular}{|c|c|c|c|}
\hline $\begin{array}{l}\text { Conhecimento das } \\
\text { Entrevistadas }\end{array}$ & $\begin{array}{c}\text { Gestantes } \\
\text { n (\%) }\end{array}$ & $\begin{array}{c}\text { Puérperas } \\
\text { n (\%) }\end{array}$ & $\begin{array}{l}\text { Valor } \\
\text { de p }\end{array}$ \\
\hline $\begin{array}{l}\text { Presente } \\
\text { Ausente }\end{array}$ & $\begin{array}{r}7(17,5 \%) \\
33(82,5 \%)\end{array}$ & $\begin{array}{r}8(20 \%) \\
32(80 \%)\end{array}$ & 0,77 \\
\hline Total & $40(100 \%)$ & $40(100 \%)$ & \\
\hline
\end{tabular}

Analisando apenas as pacientes sintomáticas (tabela 2), um maior percentual de gestantes (23.5\%) informou conhecer a respeito da fisioterapia na IUE quando comparado com as puérperas (20\%), não havendo, entretanto, diferença significativa entre os grupos.

Tabela 2 - Avaliação do conhecimento sobre fisioterapia na IUE entre mulheres sintomáticas $(\mathrm{n}=27)$.

\begin{tabular}{lccc}
$\begin{array}{l}\text { Conhecimento } \\
\text { Entrevistadas }\end{array}$ & $\begin{array}{c}\text { Gestantes } \\
\mathbf{n}(\%)\end{array}$ & $\begin{array}{c}\text { Puérperas } \\
\mathbf{n}(\%)\end{array}$ & $\begin{array}{c}\text { Valor } \\
\text { de } \boldsymbol{p}\end{array}$ \\
\hline Presente & $4(23,5 \%)$ & $2(20 \%)$ & 0,83 \\
Ausente & $13(76,5 \%)$ & $8(80 \%)$ & \\
Total & $17(100 \%)$ & $10(100 \%)$ & \\
\hline
\end{tabular}

\section{DISCUSSÃO}

A fisioterapia é indicada como tratamento de primeira escolha para a incontinência urinária de esforço desde 2005 pela Sociedade Internacional de Continência ${ }^{21}$, no entanto, existem poucos serviços públicos que prestam assistência fisioterapêutica para mulheres incontinentes no Brasil ${ }^{22}$, fato que pode contribuir para o desconhecimento sobre a mesma, o que foi verificado no presente estudo, visto que menos de $25 \%$ das mulheres que apresentaram queixas de IUE na gestação e no puerpério mencionaram saber sobre a existência desta modalidade terapêutica. É importante ressaltar também que foram encontrados poucos estudos descrevendo o perfil das mulheres portadoras de IUE que procuram tal assistência.

Embora o tratamento fisioterápico ainda seja pouco conhecido e procurado, devido ao desconforto e pudor por grande parte das mulheres em relação aos profissionais, os resultados são satisfatórios e compreendem uma alternativa não invasiva para as incontinentes, sobretudo para aquelas relutantes à intervenção cirúrgica seja por problemas clínicos ou pessoais. ${ }^{23-27}$ Contudo, é necessário destacar que a obtenção dos objetivos pré-estabelecidos e o consequente sucesso da terapêutica em questão, somente são possíveis mediante colaboração e disciplina da paciente. ${ }^{6}$

Existem relatos da eficácia do tratamento da IUE utilizando recursos fisioterápicos desde a década de 1980. Burgio, Robinson e Engel ${ }^{24}$ notaram uma redução média de $82 \%$ nos episódios de incontinência utilizando exercícios com biofeedback para reabilitação do assoalho pélvico. Segundo Oliveira e $\operatorname{Garcia}^{25}$, a cinesioterapia do assoalho pélvico representa um importante recurso terapêutico para a manutenção e/ou fortalecimento desta musculatura. Além disso, a eletroestimulação funcional dessa região tem sido usada como um componente adicional para intensificar a atividade muscular. ${ }^{26}$

Estudos recentes ratificaram a eficácia da cinesioterapia e da eletroestimulação endovaginal no ganho de força e funcionalidade do assoalho pélvico, associados à melhora da incontinência urinária $\mathrm{e}$ da qualidade de vida. ${ }^{26,27}$ Fürst e colaboradores ${ }^{28}$ demonstraram que a eletroestimulação endovaginal utilizada de forma isolada apresentou resultados semelhantes no que diz respeito às queixas de IUE quando comparada com a associação da mesma ao treinamento da musculatura do assoalho pélvico, que representa a primeira linha de tratamento conservador. ${ }^{27}$

Trabalhos conduzidos por diferentes autores sugerem que programas de exercícios terapêuticos podem ser efetivos no controle da IUE e na melhora da qualidade de vida. ${ }^{29-31}$

Técnicas como a reeducação postural global e a ginástica hipopressiva também têm se mostrado eficazes no tratamento da IUE feminina. ${ }^{32-35}$

\section{CONCLUSÃO}

A restrição usual na realização de atividades sociais, familiares, profissionais e sexuais decorrentes da IUE é amplamente descrita na literatura e pode diminuir a qualidade de vida das mulheres incontinentes, gerando, portanto, situações de isolamento social e estresse emocional, associadas ou não à sensação de inferioridade e depressão.

Foi observado desconhecimento acerca do tratamento fisioterapêutico da IUE por aproximadamente $80 \%$ das participantes do estudo. 
Considerando apenas as mulheres sintomáticas, apenas $20 \%$ das puérperas e $23.5 \%$ das gestantes responderam ter ciência sobre a existência da fisioterapia na reabilitação da incontinência, não havendo diferença significativa entre os grupos $(\mathrm{p}=0,83)$. A despeito do número reduzido da amostra, estes achados corroboram resultados descritos em pesquisas anteriores, evidenciando a necessidade de ampliação da assistência fisioterapêutica ginecológica e obstétrica nos serviços públicos, além da urgência na implementação de atividades de educação em saúde da mulher.

\section{REFERÊNCIAS}

1. Freitas F. Rotinas em Ginecologia. $5^{\mathrm{a}}$ ed. Porto Alegre: Artmed. 2006.

2. Berek JS. Tratado de Ginecologia. $14^{\mathrm{a}}$ ed. Rio de Janeiro: Guanabara Koogan. 2008.

3. Silveira JG, Silveira GPG. Ginecopatias de causa obstétrica. In: Rezende J. Obstetrícia. $9^{\mathrm{a}}$ ed. Rio de Janeiro: Guanabara Koogan. 2002.

4. Ramos JGL, Oliveira FR, Schmidt AP. Avaliação da incontinência urinária feminina. In: Freitas F. Rotinas em Ginecologia. $5^{\mathrm{a}}$ ed. Porto Alegre: Artmed. 2006. p. 168-78.

5. Frigo LF, Bordin DF, Romeiro CAP. Avaliação da frequência de incontinência urinária em jogadoras de voleibol amadoras de Santa Maria-RS. Cinergis 2015;16(4):267-270. doi: 10.17058/ cinergis.v16i5.6761.

6. Thom D. Variations in estimates of urinary incontinence prevalence in the community: effects of differences in definition, population characteristics, and study type. J Am Geriatr Soc 1998;46(4):473-80. doi: 10.1111/j.1532-5415.1998.tb02469.x

7. Dumoulin C, Morin M, Mayrand MH, Tousignant M, Abrahamowicz M. Group physiotherapy compared to individual physiotherapy to treat urinary incontinence in aging women: study protocol for a randomized controlled trial. Trials 2017;18(1):544. doi: 10.1186/s13063-017-2261-4.

8. Guarisi T, Pinto Neto AM, Osis MJ, Pedro AO, Paiva LH, Faúndes A. Urinary incontinence among climateric brazilian women: household survey. Rev Saúde Pública 2001;35:428-35. doi: 10.1590/S0034-89102001000500004.

9. Bicalho OJ, Rocha Filho MA, Faria Neto NA. Doenças neurológicas e envelhecimento: disfunções miccionais habitualmente conseqüentes. In: Bruschini H, Kano H, Damião R (ed). I Consenso Brasileiro. Incontinência urinária, uroneurologia, disfunções miccionais. São Paulo: BG Cultural. 1999. p. 55-64. 10. Higa RL, Lopes MHBM, Reis MJ. Fatores de risco para incontinência urinária na mulher. Rev Esc Enferm 2008; 42(1):187-92. doi: 10.1590/S0080-62342008000100025.

11. Lopes DBM, Praça NS. Incontinência urinária autorreferida no pós-parto: características clínicas. Rev Esc Enferm 2012;46(3):559-64. doi: 10.1590/S0080-62342012000300005.

12. Magajewski FRL, Beckhauser MT, Grott Y. Prevalência de incontinência urinária em primigestas em um hospital no sul do Brasil. Arq Cat Med 2013;42(3):54-58.
13. Sangsawang B. Risk factors for the development of stress urinary incontinence during pregnancy in primigravidae: a review of the literature. Eur J Obstet Gynecol Reprod Biol 2014;178:27-34. doi: 10.1016/j.ejogrb.2014.04.010.

14. Guarda RI, Garibam, Nohama P, Amaral VF. Tratamento conservador da incontinência urinária de esforço. Femina 2007;35(4):219-27.

15. Bernards AT, Berghmans BC, Slieker-Tem Hove MC, Staal JB, Bie RA, Hendriks EJ. Dutch guidelines for physiotherapy in patients with stress urinary incontinence: an update. Int Urogynecol J 2014;25(2):171-9. doi: 10.1007/s00192-013-2219-3.

16. Rett MT, Simões JA, Herrmann V, Gurgel MSC, Morais SS. Qualidade de vida em mulheres após tratamento da incontinência urinária de esforço com fisioterapia. Rev Bras Ginecol Obstet 2007;29(3):134-40. doi: 10.1590/S0100-72032007000300004.

17. Volkmer C, Monticelli M, Reibnitz KS, Brüggemann OM, Sperandio FF. Incontinênciaurinária feminina: revisão sistemática de estudos qualitativos. Ciênc Saúde Coletiva 2012;17(10):270315. doi: 10.1590/S1413-81232012001000019.

18. Polden M, Mantle J. Fisioterapia em Ginecologia e Obstetrícia. $2^{\mathrm{a}}$ ed. São Paulo: Santos. 2002.

19. Oliveira APV. Qualidade de vida na percepção de mulheres no climatério submetidas à fisioterapia para incontinência urinária de esforço genuína [monografia]. Muriaé: FAMINAS. 2007.

20. Moreno AL. Fisioterapia em Uroginecologia. $1^{a}$ ed. São Paulo: Manole. 2004.

21. Abrams P, Andersson KE, Brubaker L, Cardozo L, Cottenden A, Denis L, et al. Recommendations of the International Scientific Committee: evaluation and treatment of urinary incontinence, pelvic organ prolapse and fecal incontinence. Third International Consultation on Incontinence of the International Continence Society. Paris: Health Publications. 2005.

22. Figueiredo EM, Lara JO, Cruz MC, Quintão DMG, Monteiro MVC. Perfil sociodemográfico e clínico de usuárias de serviço de fisioterapia uroginecológica da rede pública. Rev Bras Fisioter 2008;12(2):136-42. doi: 10.1590/S1413-35552008000200010.

23. Ghaderi F, Oskouei AE. Physiotherapy for Women with Stress Urinary Incontinence: A Review Article. J Phys Ther Sci 2014;26(9):1493-1499.

24. Burgio KL, Robinson JC, Engel BT. The role of biofeedback in Kegel exercise training for stress urinary incontinence. Am J Obstet Gynecol 1986;154(1):58-64.

25. Oliveira JR, Garcia RR. Kinesiotherapy on treatment of Urinary Incontinences in elderly women. Rev Bras Geriatr Gerontol 2011;14(2):343-351. doi: 10.1590/S1809-98232011000200014. 26. Glisoi, SFN; Girelli, P. Importância da fisioterapia na conscientização e aprendizagem da contração da musculatura do assoalho pélvico em mulheres com incontinência urinária. Rev Bras Clín Med 2011;9(6);408-413. doi: 10.1590/S010351502012000300019.

27. Ayeleke RO, Hay-Smith EJ, Omar MI. Pelvic floor muscle training added to another active treatment versus the same active treatment alone for urinary incontinence in women. Cochrane Database Syst Rev 2015;11:1-108. doi: 10.1002/14651858. CD010551.pub3.

28. Fürst MC, Mendonça RR, Rodrigues AO, Matos LL, Pompeo AC, Bezerra CA. Long-term results of a clinical trial comparing isolated vaginal stimulation with combined treatment for women with stress incontinence. Einstein 2014;12(2):168-74. 
doi: 10.1590/S1679-45082014AO2866.

29. Prudencio CB, Nava GTA, Cardoso MA, Marreto RB, Sousa EA, Valenti VE, Barbosa AMP. Evolution of female urinary continence after physical therapy and associated factors. Int Arch Med 2014;7:24. doi: 10.1186/1755-7682-7-24.

30. Tajiri K, Huo M, Maruyama H. Effects of Co-contraction of Both Transverse Abdominal Muscle and Pelvic Floor Muscle Exercises for Stress Urinary Incontinence: A Randomized Controlled Trial. J Phys Ther Sci 2014;26(8):1161-3. doi: 10.1589/ jpts.26.1161.

31. Lacombe AC, Riccobene VM, Nogueira LA. Effectiveness of a program of therapeutic exercises on the quality of life and lumbar disability in women withStress Urinary Incontinence. J Bodyw Mov Ther 2015;19(1):82-8. doi: 10.1016/j. jbmt.2014.04.002.

32. Fozzatti MCM, Palma P, Herrmann V, Dambros M. Impacto da reeducação postural global no tratamento da incontinência urinária de esforço feminina. Rev Assoc Med Bras 2008;54(1):1722. doi: 10.1590/S0104-42302008000100015

33. Costa TF, Resende APM, Seleme MR et al. Ginástica hipopressiva como recurso proprioceptivo para os músculos do assoalho pélvico de mulheres incontinentes. Fisioter Bras 2011;12(5):365-69.

34. Valente MG, Freire AB, Real AA, Pozzebon MM, Braz MM, Hommerding PX. Efeitos da ginástica abdominal hipopressiva sobre a musculatura pélvica em mulheres incontinentes. Cinergis 2015;16(4):237-41. doi: 10.17058/cingergis.v16i5.6471.

35. Pereira AR, Côrtes MA, Valentim FCV, Pozza AM, Rocha LPO. Proposta de tratamento fisioterapêutico para melhoria da incontinência urinária de esforço pós- trauma: relato de caso. RCEAM 2014;2:10-19.

Como citar: CAIXETA NETO, Ademar Gonçalves et al. Avaliação do conhecimento sobre o tratamento fisioterápico da incontinência urinária de esforço entre gestantes e puérperas atendidas na rede pública de saúde do município de Patrocínio-MG. Revista Interdisciplinar de Promoção da Saúde, Santa Cruz do Sul, v. 1, n. 1, jan. 2018. ISSN 2595-3664. Disponível em: <https:// online.unisc.br/seer/index.php/ripsunisc/article/view/11942>. Acesso em: 01 janeiro 2018. doi:http://dx.doi.org/10.17058/rips. v1i1.11942. 